\title{
Logarithmically-concave moment measures I
}

\author{
Bo’az Klartag*
}

\begin{abstract}
We discuss a certain Riemannian metric, related to the toric Kähler-Einstein equation, that is associated in a linearly-invariant manner with a given log-concave measure in $\mathbb{R}^{n}$. We use this metric in order to bound the second derivatives of the solution to the toric KählerEinstein equation, and in order to obtain spectral-gap estimates similar to those of Payne and Weinberger.
\end{abstract}

\section{Contents}

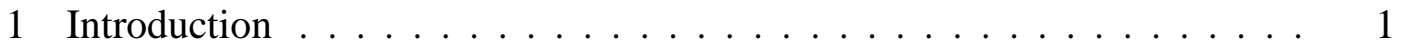

2 Continuity of the moment measure ................ 4

3 A preliminary weak bound using the maximum principle . . . . . . . . 9

4 Diffusion processes and stochastic completeness . . . . . . . . . 13

5 Bakry-Émery technique . . . . . . . . . . . . . . . 18

6 The Brascamp-Lieb inequality as a Poincaré inequality . . . . . . . 23

\section{Introduction}

In this paper we explore a certain geometric structure related to the moment measure of a convex function. This geometric structure is well-known in the community of complex geometers, see, e.g., Donaldson [13] for a discussion from the perspective of Kähler geometry.

Our motivation stems from the Kannan-Lovasź-Simonovits conjecture [16, Section 5], which is concerned with the isoperimetric problem for high-dimensional convex bodies. Essentially, our idea is to replace the standard Euclidean metric by a special Riemannian metric on the given convex body $K$. This Riemannian metric has many favorable properties, such as a Poincaré inequality with constant one, a positive Ricci tensor, the linear functions are eigenfunctions of the Laplacian, etc. Perhaps this alternative geometry does not deviate too much from the standard Euclidean geometry on $K$, and it is conceivable that the study of this Riemannian metric will turn out to be relevant to the Kannan-Lovasź-Simonovits conjecture.

Let $\mu$ be an arbitrary Borel probability measure on $\mathbb{R}^{n}$ whose barycenter is at the origin. Assume furthermore that $\mu$ is not supported in a hyperplane. It was proven in [12] that there

*School of Mathematical Sciences, Tel Aviv University, Tel Aviv 69978, Israel. E-mail: klartagb@tau.ac.il. 
exists an essentially-continuous convex function $\psi: \mathbb{R}^{n} \rightarrow \mathbb{R} \cup\{+\infty\}$, uniquely determined up to translations, such that $\mu$ is the moment measure of $\psi$, i.e.,

$$
\int_{\mathbb{R}^{n}} b(y) d \mu(y)=\int_{\mathbb{R}^{n}} b(\nabla \psi(x)) e^{-\psi(x)} d x
$$

for any $\mu$-integrable function $b: \mathbb{R}^{n} \rightarrow \mathbb{R}$. In other words, the gradient map $x \mapsto \nabla \psi(x)$ pushes the probability measure $e^{-\psi(x)} d x$ forward to $\mu$. The argument in [12] closely follows the variational approach of Berman and Berndtsson [5], which succeeded the continuity methods of Wang and Zhu [29] and Donaldson [13].

Even in the case where $\mu$ is absolutely-continuous with a $C^{\infty}$-smooth density, it is not guaranteed that $\psi$ is differentiable. From the regularity theory of the Brenier map, developed by Caffarelli [9] and Urbas [28], we learn that in order to conclude that $\psi$ is sufficiently smooth, one has to assume that the support of $\mu$ is convex.

An absolutely-continuous probability measure on $\mathbb{R}^{n}$ is called log-concave if it is supported on an open, convex set $K \subset \mathbb{R}^{n}$, and its density takes the form $\exp (-\rho)$ where the function $\rho: K \rightarrow \mathbb{R}$ is convex. An important example of a log-concave measure is the uniform probability measure on a convex body in $\mathbb{R}^{n}$. Here we assume that $\mu$ is log-concave and furthermore, we require that the following conditions are met:

(1) The convex set $K \subset \mathbb{R}^{n}$ is bounded, the function $\rho$ is $C^{\infty}$-smooth, and $\rho$ and its derivatives of all orders are bounded in $K$.

Under these regularity assumptions, we can assert that

(2) The convex function $\psi$ is finite and $C^{\infty}$-smooth in the entire $\mathbb{R}^{n}$.

The validity of (2) under the assumption (1) was proven by Wang and Zhu [29] and by Donaldson [13] via the continuity method. Berman and Berndtsson [5] explained how to deduce (2) from (1) by using Caffarelli's regularity theory [9]. In fact, the argument in [5] requires only the boundness of $\rho$, and not of its derivatives, see also the Appendix in Alesker, Dar and Milman [2]. Since the function $\psi$ is smooth, the transport equation

$$
e^{-\rho(\nabla \psi(x))} \operatorname{det} \nabla^{2} \psi(x)=e^{-\psi(x)}
$$

holds everywhere in $\mathbb{R}^{n}$, where $\nabla^{2} \psi(x)$ is the Hessian matrix of $\psi$ (see, e.g., McCann [22]). In the case where $\rho \equiv$ Const, equation (3) is called the toric Kähler-Einstein equation. We write $x \cdot y$ for the standard scalar product of $x, y \in \mathbb{R}^{n}$, and $|x|=\sqrt{x \cdot x}$.

Theorem 1.1. Let $\mu$ be a log-concave probability measure on $\mathbb{R}^{n}$ with barycenter at the origin that satisfies the regularity conditions (1). Then, with the above notation, for any $x \in \mathbb{R}^{n}$,

$$
\Delta \psi(x) \leq 2 R^{2}(K)
$$

where $R(K)=\sup _{x \in K}|x|$ is the outer radius of $K$, and $\Delta \psi$ is the Laplacian of $\psi$.

Theorem 1.1 is proven by analyzing a certain weighted Riemannian manifold. A weighted Riemannian manifold, sometimes called a Riemannian metric-measure space, is a triple

$$
X=(\Omega, g, \mu)
$$


where $\Omega$ is a smooth manifold (usually an open set in $\mathbb{R}^{n}$ ), where $g$ is a Riemannian metric on $\Omega$, and $\mu$ is a measure on $\Omega$ with a smooth density with respect to the Riemannian volume measure. In this paper we study the weighted Riemannian manifold

$$
M_{\mu}^{*}=\left(\mathbb{R}^{n}, \nabla^{2} \psi, e^{-\psi(x)} d x\right)
$$

That is, the measure associated with $M_{\mu}^{*}$ has density $e^{-\psi}$ with respect to the Lebesgue measure on $\mathbb{R}^{n}$, and the Riemannian tensor on $\mathbb{R}^{n}$ which is induced by the Hessian of $\psi$ is

$$
\sum_{i, j=1}^{n} \psi_{i j} d x^{i} d x^{j}
$$

where we abbreviate $\psi_{i j}=\partial^{2} \psi / \partial x^{i} \partial x^{j}$. There is also a dual description of $M_{\mu}^{*}$. Recall that the Legendre transform of $f: \mathbb{R}^{n} \rightarrow \mathbb{R} \cup\{+\infty\}$ is the convex function

$$
f^{*}(x)=\sup _{\substack{y \in \mathbb{R}^{n} \\ f(y)<+\infty}}[x \cdot y-f(y)] \quad\left(x \in \mathbb{R}^{n}\right) .
$$

We refer the reader to Rockafellar [26] for the basic properties of the Legendre transform. Denote $\varphi=\psi^{*}$. From (3) we see that the Hessian matrix of the convex function $\psi$ is always invertible, hence it is positive-definite. Therefore $\varphi$ is a smooth function in $K$ whose Hessian is always positive-definite. Consequently, the map $\nabla \varphi: K \rightarrow \mathbb{R}^{n}$ is a diffeomorphism, and $\nabla \psi$ is its inverse map. One may directly verify that the weighted Riemannian manifold $M_{\mu}^{*}$ is canonically isomorphic to

$$
M_{\mu}=\left(K, \nabla^{2} \varphi, \mu\right),
$$

with $x \mapsto \nabla \psi(x)$ being the isomorphism map. In differential geometry, the isomorphism between $M_{\mu}$ and $M_{\mu}^{*}$ is the passage from complex coordinates to action/angle coordinates, see, e.g., Abreu [1]. Here are some basic properties of our weighted Riemannian manifold:

(i) The space $M_{\mu}$ is stochastically complete. That is, the diffusion process associated with $M_{\mu}$ is well-defined, it has $\mu$ as a stationary measure and "it never reaches the boundary of $K$ ".

(ii) The Bakry-Émery-Ricci tensor of $M_{\mu}$ is positive. In fact, it is at least half of the Riemannian metric tensor.

(iii) The Laplacian associated with $M_{\mu}$ has an interesting spectrum: The first non-zero eigenvalue is -1 , and the corresponding eigenspace contains all linear functions.

Property (ii) is a particular case of the results of Kolesnikov [21, Theorem 4.3] (the notation of Kolesnikov is related to ours via $V=\Phi=\psi$ ), and properties (i) and (iii) are discussed below. It is important to note that the construction of $M_{\mu}$ does not rely on the Euclidean structure, and that in principle we could have replaced $\mathbb{R}^{n}$ with an abstract $n$-dimensional linear space. This is in sharp contrast with the Riemannian metric-measure space $\left(\mathbb{R}^{n},|\cdot|, \mu\right)$ that is frequently used for the analysis of the log-concave measure $\mu$.

In the following sections we prove the assertions made in the Introduction, and as a sample of possible applications, we explain below how to recover the classical Payne-Weinberger spectral gap inequality [25], up to a constant factor: 
Corollary 1.2. Let $\mu$ be a log-concave probability measure on $\mathbb{R}^{n}$ with barycenter at the origin that satisfies the regularity conditions (1). Then, for any $\mu$-integrable, smooth function $f: K \rightarrow \mathbb{R}$

$$
\int_{K} f^{2} d \mu-\left(\int_{K} f d \mu\right)^{2} \leq 2 R^{2}(K) \int_{K}|\nabla f|^{2} d \mu
$$

The constant $2 R^{2}(K)$ on the right-hand side of (6) is not optimal. In the case where $\mu$ is the uniform probability measure on a convex body $K \subset \mathbb{R}^{n}$ with a central symmetry (i.e., $K=-K)$, the best possible constant is $4 R^{2}(K) / \pi^{2}$, see Payne and Weinberger [25].

Throughout this note, a convex body in $\mathbb{R}^{n}$ is a bounded, open, convex set. We write log for the natural logarithm. A smooth function or a smooth manifold are $C^{\infty}$-smooth. The unit sphere is $S^{n-1}=\left\{x \in \mathbb{R}^{n} ;|x|=1\right\}$. The five sections below use a variety of techniques, from Itô calculus to maximum principles. We tried to make each section as independent of the others as possible.

Acknowledgements. The author would like to thank Bo Berndtsson, Dario CorderoErausquin, Ronen Eldan, Alexander Kolesnikov, Eveline Legendre, Emanuel Milman, Ron Peled, Yanir Rubinstein and Boris Tsirelson for interesting discussions related to this work. Supported by a grant from the European Research Council.

\section{Continuity of the moment measure}

This section is concerned with the continuity of the correspondence between convex functions and their moment measures. Our main result here is Proposition 2.1 below. We say that a convex function $\psi: \mathbb{R}^{n} \rightarrow \mathbb{R}$ is centered if

$$
\int_{\mathbb{R}^{n}} e^{-\psi(x)} d x=1, \quad \int_{\mathbb{R}^{n}} x_{i} e^{-\psi(x)} d x=0, i=1, \ldots, n .
$$

The role of the barycenter condition in (7) is to prevent translations of $\psi$ which result in the same moment measure. It is well-known that any convex function $\psi: \mathbb{R}^{n} \rightarrow \mathbb{R}$ satisfying $\int e^{-\psi}=1$ must tend to $+\infty$ at infinity. More precisely, for any such convex function $\psi$ there exist $A, B>0$ with

$$
\psi(x) \geq A|x|-B \quad\left(x \in \mathbb{R}^{n}\right),
$$

see, e.g., [18, Lemma 2.1]).

Proposition 2.1. Let $\Omega \subset \mathbb{R}^{n}$ be a compact set, and let $\psi, \psi_{1}, \psi_{2}, \ldots: \mathbb{R}^{n} \rightarrow \mathbb{R}$ be centered, convex functions. Denote by $\mu, \mu_{1}, \mu_{2}, \ldots$ the corresponding moment measures, which are assumed to be supported in $\Omega$. Then the following are equivalent:

(i) $\psi_{\ell} \longrightarrow \psi$ pointwise in $\mathbb{R}^{n}$.

(ii) $\mu_{\ell} \longrightarrow \mu$ weakly (i.e., $\int b d \mu_{\ell} \rightarrow \int b d \mu$ for any continuous function $b: \Omega \rightarrow \mathbb{R}$ ). 
Several lemmas are required for the proof of Proposition 2.1. For a centered, convex function $\psi: \mathbb{R}^{n} \rightarrow \mathbb{R}$ we define

$$
K(\psi)=\left\{x \in \mathbb{R}^{n} ; \psi(x) \leq 2 n+\inf _{y \in \mathbb{R}^{n}} \psi(y)\right\}
$$

a convex set in $\mathbb{R}^{n}$. Since the barycenter of $e^{-\psi(x)} d x$ lies at the origin, then $\psi(0) \leq n+$ $\inf _{x \in \mathbb{R}^{n}} \psi(x)$, according to Fradelizi [14]. Hence the origin is necessarily in the interior of $K(\psi)$. For $x \in \mathbb{R}^{n}$ consider the Minkowski functional

$$
\|x\|_{\psi}=\inf \{\lambda>0 ; x / \lambda \in K(\psi)\} .
$$

Since a convex function is continuous, then $\psi\left(x /\|x\|_{\psi}\right)=2 n+\inf \psi$ for any $0 \neq x \in \mathbb{R}^{n}$. The following lemma is well-known, but nevertheless its proof is provided for completeness.

Lemma 2.2. Let $\psi: \mathbb{R}^{n} \rightarrow \mathbb{R}$ be a centered, convex function. Then,

$$
\psi(x) \geq n\|x\|_{\psi}+\psi(0)-2 n \quad\left(x \in \mathbb{R}^{n}\right) .
$$

Proof. Since the barycenter of $e^{-\psi(x)} d x$ lies at the origin,

$$
\psi(0) \leq n+\inf _{x \in \mathbb{R}^{n}} \psi(x)
$$

Whenever $x \in K(\psi)$ we have $\|x\|_{\psi} \leq 1$. Therefore (9) follows from (10) for $x \in K(\psi)$. In order to prove (9) for $x \notin K(\psi)$, we observe that for such $x$ we have $\|x\|_{\psi} \geq 1$ and hence

$$
\psi(0)+n \leq \inf _{y \in \mathbb{R}^{n}} \psi(y)+2 n=\psi\left(\frac{x}{\|x\|_{\psi}}\right) \leq\left(1-\frac{1}{\|x\|_{\psi}}\right) \cdot \psi(0)+\frac{1}{\|x\|_{\psi}} \cdot \psi(x),
$$

due to the convexity of $\psi$. We conclude that $\psi(x) \geq \psi(0)+n\|x\|_{\psi}$ for any $x \notin K(\psi)$, and (9) is proven in all cases.

Proof of the direction (i) $\Rightarrow$ (ii) in Proposition 2.1] Denote

$$
K=\left\{x \in \mathbb{R}^{n} ; \psi(x)<2 n+1+\psi(0)\right\}
$$

an open, convex set containing the origin. Since $e^{-\psi}$ is integrable, then $K$ must be of finite volume, hence bounded. According to Rockafellar [26, Theorem 10.8], the convergence of $\psi_{\ell}$ to $\psi$ is locally uniform in $\mathbb{R}^{n}$. In particular, the convergence is uniform on $K$. Setting $M=\psi(0)-1$ we conclude that there exists $\ell_{0} \geq 1$ such that

$$
K\left(\psi_{\ell}\right) \subseteq K, \quad \psi_{\ell}(0) \geq M \quad \text { for all } \ell \geq \ell_{0} .
$$

Denote $R=\sup _{x \in K}|x|$. From (11) and Lemma2.2, for any $\ell \geq \ell_{0}$,

$$
\psi_{\ell}(x) \geq n\|x\|_{\psi_{\ell}}+\psi_{\ell}(0)-2 n \geq \frac{n}{R}|x|+(M-2 n) \quad\left(x \in \mathbb{R}^{n}\right) .
$$


According to our assumption (i) and [26, Theorem 24.5] we have that

$$
\nabla \psi_{\ell}(x) \stackrel{\ell \rightarrow \infty}{\longrightarrow} \nabla \psi(x)
$$

for any $x \in \mathbb{R}^{n}$ in which $\psi, \psi_{1}, \psi_{2}, \ldots$ are differentiable. Let $b: \Omega \rightarrow \mathbb{R}$ be a continuous function. Since a convex function is differentiable almost everywhere, we conclude that

$$
b\left(\nabla \psi_{\ell}(x)\right) e^{-\psi_{\ell}(x)} \stackrel{\ell \rightarrow \infty}{\longrightarrow} b(\nabla \psi(x)) e^{-\psi(x)} \quad \text { for almost any } x \in \mathbb{R}^{n} .
$$

The function $b$ is bounded because $\Omega$ is compact. We may use the dominated convergence theorem, thanks to (12), and conclude that

$$
\int_{\Omega} b d \mu_{\ell}=\int_{\mathbb{R}^{n}} b\left(\nabla \psi_{\ell}(x)\right) e^{-\psi_{\ell}(x)} d x \stackrel{\ell \rightarrow \infty}{\longrightarrow} \int_{\mathbb{R}^{n}} b(\nabla \psi(x)) e^{-\psi(x)} d x=\int_{\Omega} b d \mu .
$$

Thus (ii) is proven.

It still remains to prove the direction (ii) $\Rightarrow$ (i) in Proposition 2.1 A function $f: \mathbb{R}^{n} \rightarrow$ $\mathbb{R}$ is $L$-Lipschitz if $|f(x)-f(y)| \leq L|x-y|$ for any $x, y \in \mathbb{R}^{n}$.

Lemma 2.3. Let $L, \varepsilon>0$. Suppose that $\psi: \mathbb{R}^{n} \rightarrow \mathbb{R}$ is a centered, L-Lipschitz, convex function, such that

$$
\int_{\mathbb{R}^{n}}|\nabla \psi(x) \cdot \theta| e^{-\psi(x)} d x \geq \varepsilon \quad \text { for all } \theta \in S^{n-1} .
$$

Then,

$$
\alpha|x|-\beta \leq \psi(x) \leq L|x|+\gamma \quad\left(x \in \mathbb{R}^{n}\right),
$$

where $\alpha, \beta, \gamma>0$ are constants depending only on $L, \varepsilon$ and $n$.

Proof. Fix $\theta \in S^{n-1}$ and set $H=\theta^{\perp}$, the hyperplane orthogonal to $\theta$. The function

$$
m_{\theta}(y)=\inf _{t \in \mathbb{R}} \psi(y+t \theta) \quad(y \in H)
$$

is convex. Furthermore, for any fixed $y \in H$, the function $t \mapsto \psi(y+t \theta)$ is convex, $L$-Lipschitz and tends to $+\infty$ as $t \rightarrow \pm \infty$. Hence the one-dimensional convex function $t \mapsto \psi(y+t \theta)$ attains its minimum at a certain point $t_{0} \in \mathbb{R}$, is non-decreasing on $\left[t_{0},+\infty\right)$ and non-increasing on $\left(-\infty, t_{0}\right]$. Therefore, for any $y \in H$,

$$
\int_{-\infty}^{\infty}\left|\frac{\partial \psi(y+t \theta)}{\partial t}\right| e^{-\psi(y+t \theta)} d t=\int_{-\infty}^{\infty}\left|\frac{\partial}{\partial t} e^{-\psi(y+t \theta)}\right| d t=2 e^{-m_{\theta}(y)} .
$$

We now integrate over $y \in H$ and use Fubini's theorem to conclude that

$$
\int_{\mathbb{R}^{n}}|\nabla \psi(x) \cdot \theta| e^{-\psi(x)} d x=2 \int_{H} e^{-m_{\theta}(y)} d y .
$$

Consider the interval

$$
I_{\theta}=\{t \in \mathbb{R} ; t \theta \in K(\psi)\}
$$


Then,

$$
\int_{-\infty}^{\infty} e^{-\psi(t \theta) / 2} d t \geq \int_{I_{\theta}} e^{-\psi(t \theta) / 2} d t \geq e^{-n-\frac{m_{\theta}(0)}{2}}\left|I_{\theta}\right|
$$

where $\left|I_{\theta}\right|$ is the length of the interval $I_{\theta}$. Fix a point $y \in H$. Then there exists $t_{0} \in \mathbb{R}$ for which $m_{\theta}(y)=\psi\left(y+t_{0} \theta\right)$. From (17) and from the convexity of $\psi$,

$$
\begin{aligned}
\int_{-\infty}^{\infty} e^{-\psi\left(\frac{y}{2}+t \theta\right)} d t & =\frac{1}{2} \int_{-\infty}^{\infty} e^{-\psi\left(\frac{y+t_{0} \theta}{2}+\frac{t \theta}{2}\right)} d t \geq \frac{1}{2} e^{-\frac{m_{\theta}(y)}{2}} \int_{-\infty}^{\infty} e^{-\frac{\psi(t \theta)}{2}} d t \\
& \geq \frac{1}{2} e^{-\frac{m_{\theta}(y)+m_{\theta}(0)}{2}} e^{-n}\left|I_{\theta}\right| \geq \frac{1}{2} e^{-m_{\theta}(y)} e^{-2 n}\left|I_{\theta}\right|
\end{aligned}
$$

where in the last passage we used the fact that $m_{\theta}(0) \leq \psi(0) \leq n+\inf \psi \leq n+m_{\theta}(y)$, because the barycenter of $e^{-\psi(x)} d x$ lies at the origin. Integrating (18) over $y \in H$, we see that

$$
\int_{H} e^{-m_{\theta}(y)} d y \leq \frac{2 e^{2 n}}{\left|I_{\theta}\right|} \int_{H} \int_{-\infty}^{\infty} e^{-\psi\left(\frac{y}{2}+t \theta\right)} d t d y=\frac{2^{n} e^{2 n}}{\left|I_{\theta}\right|} \int_{\mathbb{R}^{n}} e^{-\psi}=\frac{2^{n} e^{2 n}}{\left|I_{\theta}\right|}
$$

Combine the last inequality with (13) and (15). This leads to the bound

$$
\left|I_{\theta}\right| \leq C_{n}\left(\int_{\mathbb{R}^{n}}|\nabla \psi(x) \cdot \theta| e^{-\psi(x)} d x\right)^{-1} \leq \frac{C_{n}}{\varepsilon},
$$

for some constant $C_{n}$ depending only on $n$. Recall that the origin belongs to $K(\psi)$ and hence $0 \in I_{\theta}$. By letting $\theta$ range over all of $S^{n-1}$ and glancing at (16) and (19), we see that

$$
K(\psi) \subseteq B\left(0, C_{n} / \varepsilon\right)
$$

where $B(x, r)=\left\{y \in \mathbb{R}^{n} ;|y-x| \leq r\right\}$. From (20) and from Lemma 2.2.

$$
\psi(x) \geq \psi(0)-2 n+n\|x\|_{\psi} \geq \psi(0)-2 n+\frac{\varepsilon}{\tilde{C}_{n}}|x| \quad\left(x \in \mathbb{R}^{n}\right),
$$

for $\tilde{C}_{n}=C_{n} / n$. By integrating (21) we obtain

$$
1=\int_{\mathbb{R}^{n}} e^{-\psi} \leq e^{-(\psi(0)-2 n)} \int_{\mathbb{R}^{n}} e^{-\varepsilon|x| / \tilde{C}_{n}} d x .
$$

Therefore, $\psi(0) \leq \gamma$ for $\gamma=2 n+\log \left(\int_{\mathbb{R}^{n}} e^{-\varepsilon|x| / \tilde{C}_{n}} d x\right)$. Since $\psi$ is $L$-Lipschitz, then the right-hand side inequality of (14) follows. Next, observe that

$$
1=\int_{\mathbb{R}^{n}} e^{-\psi(x)} d x \geq \int_{\mathbb{R}^{n}} e^{-\psi(0)-L|x|} d x=e^{-\psi(0)} \int_{\mathbb{R}^{n}} e^{-L|x|} d x .
$$

Hence $\psi(0) \geq \log \left(\int_{\mathbb{R}^{n}} e^{-L|x|} d x\right)$, and the left-hand side inequality of (14) follows from (21). 
Proof of the direction (ii) $\Rightarrow$ (i) in Proposition 2.1

Step 1. We claim that

$$
\liminf _{\ell \rightarrow \infty}\left(\inf _{\theta \in S^{n-1}} \int_{\Omega}|x \cdot \theta| d \mu_{\ell}(x)\right)>0
$$

Assume that (22) fails. Then there exist sequences $\ell_{j} \in \mathbb{N}$ and $\theta_{j} \in S^{n-1}$ such that

$$
\lim _{j \rightarrow \infty} \int_{\Omega}\left|x \cdot \theta_{j}\right| d \mu_{\ell_{j}}(x)=0
$$

Passing to a subsequence, if necessary, we may assume that $\theta_{j} \longrightarrow \theta_{0} \in S^{n-1}$. The sequence of functions $\left|x \cdot \theta_{j}\right|$ tends to $\left|x \cdot \theta_{0}\right|$ uniformly in $x \in \Omega$. Hence, from (ii) and (23),

$$
\int_{\Omega}\left|x \cdot \theta_{0}\right| d \mu(x)=\lim _{j \rightarrow \infty} \int_{\Omega}\left|x \cdot \theta_{0}\right| d \mu_{\ell_{j}}(x)=\lim _{j \rightarrow \infty} \int_{\Omega}\left|x \cdot \theta_{j}\right| d \mu_{\ell_{j}}(x)=0
$$

Therefore $\mu$ is supported in the hyperplane $\theta_{0}^{\perp}$. However, $\mu$ is the moment measure of the convex function $\psi: \mathbb{R}^{n} \rightarrow \mathbb{R}$, and according to [12, Proposition 1], it cannot be supported in a hyperplane. We have thus arrived at a contradiction, and (22) is proven.

Step 2. We will prove that there exist $\alpha, \beta, \gamma>0$ and $\ell_{0} \geq 1$ such that

$$
\alpha|x|-\beta \leq \psi_{\ell}(x) \leq L|x|+\gamma \quad\left(\ell \geq \ell_{0}, x \in \mathbb{R}^{n}\right) .
$$

Indeed, according to Step 1 , there exists $\ell_{0} \geq 1$ and $\varepsilon_{0}>0$ such that

$$
\int_{\mathbb{R}^{n}}\left|\nabla \psi_{\ell}(x) \cdot \theta\right| e^{-\psi_{\ell}(x)} d x=\int_{\Omega}|x \cdot \theta| d \mu_{\ell}(x)>\varepsilon_{0} \quad\left(\ell \geq \ell_{0}, \theta \in S^{n-1}\right) .
$$

Denote $L=\sup _{x \in \Omega}|x|$. The function $\psi_{\ell}$ is centered and convex. Furthermore, for almost any $x \in \mathbb{R}^{n}$ we know that $\nabla \psi_{\ell}(x) \in \Omega$, because the moment measure of $\psi_{\ell}$ is supported in $\Omega$. Hence, for $\ell \geq 1$,

$$
\left|\nabla \psi_{\ell}(x)\right| \leq L \quad \text { for almost any } x \in \mathbb{R}^{n} .
$$

Since a convex function is always locally-Lipschitz, then (26) implies that $\psi_{\ell}$ is $L$-Lipschitz, for any $\ell$. We may now apply Lemma 2.3, thanks to (25), and conclude (24).

Step 3. Assume by contradiction that there exists $x_{0} \in \mathbb{R}^{n}$ for which $\psi_{\ell}\left(x_{0}\right)$ does not converge to $\psi\left(x_{0}\right)$. Then there exist $\varepsilon>0$ and a subsequence $\ell_{j}$ such that

$$
\left|\psi_{\ell_{j}}\left(x_{0}\right)-\psi\left(x_{0}\right)\right| \geq \varepsilon \quad(j=1,2, \ldots) .
$$

From (24) we know that the sequence of functions $\left\{\psi_{\ell_{j}}\right\}_{j=1,2, \ldots}$ is uniformly bounded on any compact subset of $\mathbb{R}^{n}$. Furthermore, $\psi_{\ell_{j}}$ is $L$-Lipschitz for any $j$. According to the Arzelá-Ascoli theorem, we may pass to a subsequence and assume that $\psi_{\ell_{j}}$ converges locally uniformly in $\mathbb{R}^{n}$, to a certain function $F$. The function $F$ is convex and $L$-Lipschitz, as it is the limit of convex and $L$-Lipschitz functions. Furthermore, thanks to (24) we may apply the dominated convergence theorem and conclude that $F$ is centered. 
To summarize, the functions $F, \psi_{\ell_{1}}, \psi_{\ell_{2}}, \ldots$ are $L$-Lipschitz, centered and convex. We know that $\psi_{\ell_{j}} \longrightarrow F$ locally uniformly in $\mathbb{R}^{n}$. According to the implication (i) $\Rightarrow$ (ii) proven above, we know that $\mu_{\ell_{j}}$ converges weakly to the moment measure of $F$. But we assumed that $\mu_{\ell_{j}}$ converges weakly to $\mu$, and hence $\mu$ is the moment measure of $F$. Thus $\psi, F: \mathbb{R}^{n} \rightarrow \mathbb{R}$ are two centered, convex functions with the same moment measure $\mu$. This means that $\psi \equiv F$, according to the uniqueness part in [12]. Therefore $\psi_{\ell_{j}} \longrightarrow \psi$ pointwise in $\mathbb{R}^{n}$, in contradiction to (27), and the proof is complete.

\section{A preliminary weak bound using the maximum prin- ciple}

In this section we prove a rather weak form of Theorem 1.1, which will be needed for the proof of the theorem later on in Section 5 . Throughout this section, $\mu$ is a log-concave probability measure on $\mathbb{R}^{n}$ with barycenter at the origin, supported on a convex body $K \subset \mathbb{R}^{n}$, with density $e^{-\rho}$ satisfying the regularity conditions (1). Also, $\psi: \mathbb{R}^{n} \rightarrow \mathbb{R}$ is the smooth, convex function whose moment measure is $\mu$, which is uniquely defined up to translation, and $\varphi=\psi^{*}$ is its Legendre transform. In this section we make the following strict-convexity assumptions:

( $\star$ The convex body $K$ has a smooth boundary and its Gauss curvature is positive everywhere. Additionally, there exists $\varepsilon_{0}>0$ with

$$
\nabla^{2} \rho(x) \geq \varepsilon_{0} \cdot I d \quad(x \in K),
$$

in the sense of symmetric matrices.

Denote by $\|A\|$ the operator norm of the matrix $A$. Our goal in this section is to prove the following:

Proposition 3.1. Under the above assumptions,

$$
\sup _{x \in \mathbb{R}^{n}}\left\|\nabla^{2} \psi(x)\right\|<+\infty
$$

The argument we present for the demonstration of Proposition 3.1 closely follows the proof of Caffarelli's contraction theorem [10, Theorem 11]. An alternative approach to Proposition 3.1] is outlined in Kolesnikov [20, Section 6]. We begin the proof of Proposition 3.1 with the following lemma, which is due to Berman and Berndtsson [5]. Their proof is reproduced here for completeness.

Lemma 3.2. $\sup _{x \in K} \varphi(x)<+\infty$.

Proof. Since $K$ is bounded, it suffices to show that $\varphi$ is $\alpha$-Hölder for some $\alpha>0$. According to the Sobolev inequality in the convex domain $K \subset \mathbb{R}^{n}$ (see, e.g., [27, Chapter 1]), it is sufficient to prove that

$$
\int_{K}|\nabla \varphi(x)|^{p} d x<+\infty
$$


for some $p>n$. Fix $p>n$. The map $x \mapsto \nabla \varphi(x)$ pushes the measure $\mu$ forward to $\exp (-\psi(x)) d x$. Hence,

$$
\int_{K}|\nabla \varphi|^{p} d \mu=\int_{\mathbb{R}^{n}}|x|^{p} e^{-\psi(x)} d x<+\infty
$$

where we used the fact that $e^{-\psi}$ decays exponentially at infinity (see, e.g., (8) above or [18, Lemma 2.1]). Since $\rho$ is a bounded function on $K$ and $e^{-\rho}$ is the density of $\mu$, then 29) follows from (30).

For $x \in \mathbb{R}^{n}$ denote $h_{K}(x)=\sup _{y \in K} x \cdot y$, the supporting functional of $K$. The following lemma is analogous to [10, Lemma 4].

Lemma 3.3. $\lim _{R \rightarrow \infty} \sup _{|x| \geq R}\left|\nabla \psi(x)-\nabla h_{K}(x)\right|=0$.

Proof. The function $\varphi: K \rightarrow \mathbb{R}$ is convex, hence bounded from below by some affine function, which in turn is greater than some constant on the bounded set $K$. According to Lemma 3.2 the function $\varphi$ is also bounded from above. Set $M=\sup _{x \in K}|\varphi(x)|$. By elementary properties of the Legendre transform, for any $x \in \mathbb{R}^{n}$,

$$
\psi(x)=x \cdot \nabla \psi(x)-\varphi(\nabla \psi(x)) \leq x \cdot \nabla \psi(x)+M .
$$

However, for any $x \in \mathbb{R}^{n}$,

$$
\psi(x)=\sup _{y \in K}[x \cdot y-\varphi(y)] \geq-M+\sup _{y \in K} x \cdot y=-M+x \cdot \nabla h_{K}(x),
$$

as $\nabla h_{K}(x) \in \partial K$ is the unique point at which $\sup _{y \in K} x \cdot y$ is attained. Using (31) and (32),

$$
\left(\nabla h_{K}(x)-\nabla \psi(x)\right) \cdot \frac{x}{|x|} \leq \frac{2 M}{|x|} \quad\left(0 \neq x \in \mathbb{R}^{n}\right) .
$$

Recall that $\nabla \psi(x) \in K$ for any $x \in \mathbb{R}^{n}$. Since $\partial K$ is smooth with positive Gauss curvature, inequality (33) implies that there exist $R_{K}, \alpha_{K}>0$, depending only on $K$, with

$$
\left|\nabla h_{K}(x)-\nabla \psi(x)\right| \leq \alpha_{K} \sqrt{\frac{2 M}{|x|}} \quad \text { for }|x| \geq R_{K} .
$$

The lemma follows from (34).

For $\varepsilon>0, \theta \in \mathbb{R}^{n}$ and a function $f: \mathbb{R}^{n} \rightarrow \mathbb{R}$ denote

$$
\delta_{\theta \theta}^{(\varepsilon)} f(x)=f(x+\varepsilon \theta)+f(x-\varepsilon \theta)-2 f(x) \quad\left(x \in \mathbb{R}^{n}\right) .
$$

For a smooth $f$ and a small $\varepsilon$, the quantity $\delta_{\theta \theta}^{(\varepsilon)} f(x) / \varepsilon^{2}$ approximates the pure second derivative $f_{\theta \theta}(x)$. We would like to use the maximum principle for the function $\psi_{\theta \theta}(x)$, but we do not know whether or not it attains its supremum. This is the reason for using the approximate second derivative $\delta_{\theta \theta}^{(\varepsilon)} \psi(x)$ as a substitute. 
Corollary 3.4. Fix $0<\varepsilon<1$. Then the supremum of $\delta_{\theta \theta}^{(\varepsilon)} \psi(x)$ over all $x \in \mathbb{R}^{n}$ and $\theta \in S^{n-1}$ is attained.

Proof. According to Lemma 3.3 and the continuity and 0-homogeneity of $\nabla h_{K}(x)$,

$$
\begin{aligned}
& \lim _{R \rightarrow \infty} \sup _{\substack{|x| \geq R \\
x_{1}, x_{2} \in B(x, 1)}}\left|\nabla \psi\left(x_{1}\right)-\nabla \psi\left(x_{2}\right)\right|=\lim _{R \rightarrow \infty} \sup _{\substack{|x| \geq R \\
\text { an } \\
x_{1}, x_{2} \in B(x, 1)}}\left|\nabla h_{K}\left(x_{1}\right)-\nabla h_{K}\left(x_{2}\right)\right| \\
& =\lim _{R \rightarrow \infty} \sup _{\substack{|x|=1 \\
x_{1}, x_{2} \in B(x, 1 / R)}}\left|\nabla h_{K}\left(x_{1}\right)-\nabla h_{K}\left(x_{2}\right)\right|=0
\end{aligned}
$$

where $B(x, r)=\left\{y \in \mathbb{R}^{n} ;|x-y|<r\right\}$. From Lagrange's mean value theorem,

$$
\begin{aligned}
\delta_{\theta \theta}^{(\varepsilon)} \psi(x) & =(\psi(x+\varepsilon \theta)-\psi(x))-(\psi(x)-\psi(x-\varepsilon \theta)) \\
& \leq \varepsilon \sup _{x_{1}, x_{2} \in B(x, \varepsilon)}\left|\nabla \psi\left(x_{1}\right)-\nabla \psi\left(x_{2}\right)\right| .
\end{aligned}
$$

According to (35) and (36),

$$
\lim _{R \rightarrow \infty} \sup _{\substack{|x| \geq R \\ \theta \in S^{n}-1}} \delta_{\theta \theta}^{(\varepsilon)} \psi(x) \leq \varepsilon \lim _{R \rightarrow \infty} \sup _{\substack{|x| \geq R \\ x_{1}, x_{2} \in B(x, \varepsilon)}}\left|\nabla \psi\left(x_{1}\right)-\nabla \psi\left(x_{2}\right)\right|=0 .
$$

Since $\psi$ is convex and smooth, then the function $\delta_{\theta \theta}^{(\varepsilon)} \psi$ is non-negative and continuous in $(x, \theta) \in \mathbb{R}^{n} \times S^{n-1}$. It thus follows from (37) that its supremum is attained.

We shall apply the well-known matrix inequality, which states that when $A$ and $B$ are symmetric, positive-definite $n \times n$ matrices, then

$$
\log \operatorname{det} B \leq \log \operatorname{det} A+\operatorname{Tr}\left[A^{-1}(B-A)\right]=\log \operatorname{det} A+\operatorname{Tr}\left[A^{-1} B\right]-n,
$$

where $\operatorname{Tr}(A)$ stands for the trace of the matrix $A$. Recall that the transport equation (3) is valid, hence,

$$
\log \operatorname{det} \nabla^{2} \psi(x)=-\psi(x)+(\rho \circ \nabla \psi)(x) \quad\left(x \in \mathbb{R}^{n}\right) .
$$

In particular, $\nabla^{2} \psi(x)$ is always an invertible matrix which is in fact positive-definite. We denote its inverse by $\left(\nabla^{2} \psi(x)\right)^{-1}=\left(\psi^{i j}(x)\right)_{i, j=1, \ldots, n}$. For a smooth function $u: \mathbb{R}^{n} \rightarrow \mathbb{R}$ denote

$$
A u(x)=\operatorname{Tr}\left[\left(\nabla^{2} \psi(x)\right)^{-1} \nabla^{2} u(x)\right]=\psi^{i j}(x) u_{i j}(x) \quad\left(x \in \mathbb{R}^{n}\right),
$$

where we adhere to the Einstein convention: When an index is repeated twice in an expression, once as a subscript and once as a superscript, then we sum over this index from 1 to $n$. According to (38) for any $\theta \in \mathbb{R}^{n}$,

$$
\log \operatorname{det} \nabla^{2} \psi(x+\theta) \leq \log \operatorname{det} \nabla^{2} \psi(x)+\psi^{i j}(x) \psi_{i j}(x+\theta)-n \quad\left(x \in \mathbb{R}^{n}\right),
$$

with an equality for $\theta=0$. 
Proof of Proposition 3.1 We follow Caffarelli's argument [10, Theorem 11]. Our assumption (28) yields that the function $\rho(x)-\varepsilon_{0}|x|^{2} / 2$ is convex. Hence, for any $x, y$ such that $x-y, x+y, x \in K$,

$$
\rho(x+y)+\rho(x-y)-2 \rho(x) \geq \frac{\varepsilon_{0}}{2}\left(|x+y|^{2}+|x-y|^{2}-2|x|^{2}\right)=\varepsilon_{0}|y|^{2} .
$$

Fix $0<\varepsilon<1$ and abbreviate $\delta_{\theta \theta} f=\delta_{\theta \theta}^{(\varepsilon)} f$. From (39) and (41) as well as some simple algebraic manipulations, for any $\theta \in \mathbb{R}^{n}$,

$$
A\left(\delta_{\theta \theta} \psi\right) \geq \delta_{\theta \theta}\left(\log \operatorname{det} \nabla^{2} \psi\right)=-\delta_{\theta \theta} \psi+\delta_{\theta \theta}(\rho \circ \nabla \psi) .
$$

According to Corollary 3.4 the maximum of $(x, \theta) \mapsto \delta_{\theta \theta} \psi(x)$ over $\mathbb{R}^{n} \times S^{n-1}$ is attained at some $\left(x_{0}, e\right) \in \mathbb{R}^{n} \times S^{n-1}$. Since $\psi$ is smooth, then at the point $x_{0}$,

$$
0=\nabla\left(\delta_{e e} \psi\right)\left(x_{0}\right)=\nabla \psi\left(x_{0}+\varepsilon e\right)+\nabla \psi\left(x_{0}+\varepsilon e\right)-2 \nabla \psi\left(x_{0}\right) .
$$

In other words, there exists a vector $u \in \mathbb{R}^{n}$ such that

$$
\nabla \psi\left(x_{0}+\varepsilon e\right)=\nabla \psi\left(x_{0}\right)+u, \quad \nabla \psi\left(x_{0}-\varepsilon e\right)=\nabla \psi\left(x_{0}\right)-u .
$$

Setting $v=\nabla \psi\left(x_{0}\right)$ and using (42), we obtain

$$
\delta_{e e}(\rho \circ \nabla \psi)\left(x_{0}\right)=\rho(v+u)+\rho(v-u)-2 \rho(v) \geq \varepsilon_{0}|u|^{2} .
$$

The smooth function $x \mapsto \delta_{e e} \psi(x)$ reaches a maximum at $x_{0}$, hence the matrix $\nabla^{2}\left(\delta_{e e} \psi\right)\left(x_{0}\right)$ is negative semi-definite. Since the matrix $\left(\nabla^{2} \psi\right)^{-1}\left(x_{0}\right)$ is positive-definite, then from the definition (40),

$$
0 \geq A\left(\delta_{e e} \psi\right)\left(x_{0}\right)
$$

Now, (43), (44) and (45) yield

$$
\delta_{e e} \psi\left(x_{0}\right) \geq \delta_{e e}(\rho \circ \nabla \psi)\left(x_{0}\right) \geq \varepsilon_{0}|u|^{2} .
$$

By the convexity of $\psi$,

$$
\psi\left(x_{0}+\varepsilon e\right)-\psi\left(x_{0}\right) \leq \nabla \psi\left(x_{0}+\varepsilon e\right) \cdot(\varepsilon e)=(v+u) \cdot(\varepsilon e)
$$

and

$$
\psi\left(x_{0}-\varepsilon e\right)-\psi\left(x_{0}\right) \leq \nabla \psi\left(x_{0}-\varepsilon e\right) \cdot(-\varepsilon e)=(v-u) \cdot(-\varepsilon e) .
$$

Summing the last two inequalities yields

$$
\delta_{e e} \psi\left(x_{0}\right) \leq(v+u) \cdot(\varepsilon e)+(v-u) \cdot(-\varepsilon e)=2 \varepsilon(u \cdot e) \leq 2|u| \varepsilon .
$$

The inequalities (46) and (47) imply that $|u| \leq 2 \varepsilon / \varepsilon_{0}$ and hence from (47),

$$
\delta_{e e}(\psi)\left(x_{0}\right) \leq 4 \varepsilon^{2} / \varepsilon_{0} .
$$

Consequently, for any $x \in \mathbb{R}^{n}$ and $\theta \in S^{n-1}$ we have $\delta_{\theta \theta}^{(\varepsilon)} \psi(x) \leq 4 \varepsilon^{2} / \varepsilon_{0}$, and hence

$$
\psi_{\theta \theta}(x)=\lim _{\varepsilon \rightarrow 0^{+}} \frac{\delta_{\theta \theta}^{(\varepsilon)} \psi(x)}{\varepsilon^{2}} \leq \frac{4}{\varepsilon_{0}} .
$$

Therefore $\left\|\nabla^{2} \psi(x)\right\| \leq 4 / \varepsilon_{0}$ for any $x \in \mathbb{R}^{n}$, and the proof is complete. 
Remark 3.5. Our proof of Proposition 3.1 provides the explicit bound

$$
\sup _{x \in \mathbb{R}^{n}}\left\|\nabla^{2} \psi(x)\right\| \leq 4 / \varepsilon_{0}
$$

By arguing as in [11], one may improve the right-hand side of (48) to just $1 / \varepsilon_{0}$. We omit the straightforward details.

\section{Diffusion processes and stochastic completeness}

In this section we consider a diffusion process associated with transportation of measure. Our point of view owes much to the article by Kolesnikov [21], and we make an effort to maintain a discussion as general as the one in Kolesnikov's work.

Let $\mu$ be a probability measure supported on an open set $K \subseteq \mathbb{R}^{n}$, with density $e^{-\rho}$ where $\rho: K \rightarrow \mathbb{R}$ is a smooth function. Let $\psi: \mathbb{R}^{n} \rightarrow \mathbb{R}$ be a smooth, convex function with

$$
\lim _{R \rightarrow \infty}\left(\inf _{|x| \geq R} \psi(x)\right)=+\infty \text {. }
$$

Condition (49) holds automatically when $\int e^{-\psi}<\infty$, see (8) above. Rather than requiring that the transport equation (3) hold true, in this section we make the more general assumption that

$$
e^{-\rho(\nabla \psi(x))} \operatorname{det} \nabla^{2} \psi(x)=e^{-V(x)} \quad\left(x \in \mathbb{R}^{n}\right)
$$

for a certain smooth function $V: \mathbb{R}^{n} \rightarrow \mathbb{R}$. Clearly, when $\mu$ is the moment measure of $\psi$, equation (50) holds true with $V=\psi$ and condition (49) holds as well. The transport equation (50) means that the map $x \mapsto \nabla \psi(x)$ pushes the probability measure $e^{-V(x)} d x$ forward to $\mu$. In this section we explain and prove the following:

Proposition 4.1. Let $K \subseteq \mathbb{R}^{n}$ be an open set, and let $V, \psi: \mathbb{R}^{n} \rightarrow \mathbb{R}$ and $\rho: K \rightarrow \mathbb{R}$ be smooth functions with $\psi$ being convex. Assume (49) and (50), and furthermore, that

$$
\inf _{x \in K} \nabla \rho(x) \cdot x>-\infty
$$

Then the weighted Riemannian manifold $M=\left(\mathbb{R}^{n}, \nabla^{2} \psi, e^{-V(x)} d x\right)$ is stochastically complete.

Remark 4.2. Note that in the most interesting case where $V=\psi$, the weighted Riemannian manifold $M$ from Proposition 4.1 coincides with $M_{\mu}^{*}$ as defined in (4) and (5) above. Additionally, in the case where $\mu$ is log-concave with barycenter at the origin, condition (51) does hold true: In this case, according to Fradelizi [14], we know that $\rho(0) \leq n+\inf _{x \in K} \rho(x)$. By convexity,

$$
\nabla \rho(x) \cdot x \geq \rho(x)-\rho(0) \geq-n \quad(x \in K),
$$

and (51) follows. Thus Proposition 4.1 implies the stochastic completeness of $M_{\mu}^{*}$ when $\mu$ is a log-concave probability measure with barycenter at the origin, which satisfies the regularity conditions (1). 
We now turn to a detailed explanation of stochastic completeness of a weighted Riemannian manifold. See, e.g., Grigor'yan [15] for more information. The Dirichlet form associated with the weighted Riemannian manifold $M=(\Omega, g, \nu)$ is defined as

$$
\Gamma(u, v)=\int_{\Omega} g\left(\nabla_{g} u, \nabla_{g} v\right) d \nu,
$$

where $u, v: \Omega \rightarrow \mathbb{R}$ are smooth functions for which the integral in (52) exists. Here, $\nabla_{g} u$ stands for the Riemannian gradient of $u$. The Laplacian associated with $M$ is the unique operator $L$, acting on smooth functions $u: \Omega \rightarrow \mathbb{R}$, for which

$$
\int_{\Omega}(L u) v d \nu=-\Gamma(u, v)
$$

for any compactly-supported, smooth function $v: \Omega \rightarrow \mathbb{R}$. In the case of the weighted manifold $M=\left(\mathbb{R}^{n}, \nabla^{2} \psi, e^{-V(x)} d x\right)$ from Proposition 4.1, we may express the Dirichlet form as follows:

$$
\Gamma(u, v)=\int_{\mathbb{R}^{n}}\left(\psi^{i j} u_{i} v_{j}\right) e^{-V}
$$

where $\nabla^{2} \psi(x)^{-1}=\left(\psi^{i j}(x)\right)_{i, j=1, \ldots, n}$ and $u_{i}=\partial u / \partial x^{i}$. Note that the matrix $\nabla^{2} \psi(x)$ is invertible, thanks to (50). As in Section 3 above, we use the Einstein summation convention; thus in (54) we sum over $i, j$ from 1 to $n$. We will also make use of abbreviations such as $\psi_{i j k}=\partial^{3} \psi /\left(\partial x^{i} \partial x^{j} \partial x^{k}\right)$, and also $\psi_{j \ell}^{i}=\psi^{i k} \psi_{j k \ell}$ and $\psi_{k}^{i j}=\psi^{i \ell} \psi^{j m} \psi_{\ell m k}$. Therefore, for example,

$$
\left(\psi^{i j}\right)_{k}=\frac{\partial \psi^{i j}(x)}{\partial x^{k}}=-\psi^{i \ell} \psi^{j m} \psi_{\ell m k}=-\psi_{k}^{i j}
$$

We may now express the Laplacian $L$ associated with $M=\left(\mathbb{R}^{n}, \nabla^{2} \psi, e^{-V(x)} d x\right)$ by

$$
L u=\psi^{i j} u_{i j}-\left(\psi_{j}^{i j}+\psi^{i j} V_{j}\right) u_{i}
$$

as may be directly verified from (54) by integration by parts.

Lemma 4.3. For any smooth function $u: \mathbb{R}^{n} \rightarrow \mathbb{R}$,

$$
L u=\psi^{i j} u_{i j}-\sum_{j=1}^{n} \rho_{j}(\nabla \psi(x)) u_{j}
$$

Proof. We take the logarithmic derivative of (50) and obtain that for $\ell=1, \ldots, n$,

$$
\psi_{i \ell}^{i}(x)=-V_{\ell}(x)+\sum_{i=1}^{n} \rho_{i}(\nabla \psi(x)) \psi_{i \ell}(x) \quad\left(x \in \mathbb{R}^{n}\right) .
$$

Multiplying (57) by $\psi^{j \ell}$ and summing over $\ell$ we see that for $j=1, \ldots, n$,

$$
\psi_{i}^{i j}(x)=-\psi^{j \ell}(x) V_{\ell}(x)+\rho_{j}(\nabla \psi(x)) \quad\left(x \in \mathbb{R}^{n}\right) .
$$

Now (56) follows from (55) and (58). 
Lemma 4.4. Under the assumptions of Proposition 4.1 there exists $A \geq 0$ such that for all $x \in \mathbb{R}^{n}$,

$$
(L \psi)(x) \leq A
$$

Proof. Set $A=\max \left\{0, n-\inf _{y \in K} \nabla \rho(y) \cdot y\right\}$, which is a finite number according to our assumption (51). From Lemma4.3.

$$
L \psi(x)=\psi^{i j} \psi_{i j}-\sum_{j=1}^{n} \rho_{j}(\nabla \psi(x)) \psi_{j}(x)=n-\sum_{j=1}^{n} \rho_{j}(\nabla \psi(x)) \psi_{j}(x) .
$$

It remains to prove that $n-\sum_{j} \rho_{j}(\nabla \psi(x)) \psi_{j}(x) \leq A$, or equivalently, we need to show that

$$
\nabla \rho(y) \cdot y \geq n-A \quad \text { for all } y \in K
$$

However, (59) holds true in view of the definition of $A$ above. Therefore $L \psi \leq A$ pointwise in $\mathbb{R}^{n}$.

The Laplacian $L$ associated with a weighted Riemannian manifold $M$ is a second-order, elliptic operator with smooth coefficients. We say that $M$ is stochastically complete if the Itô diffusion process whose generator is $L$ is well-defined at all times $t \in[0, \infty)$. In the particular case of Proposition 4.1, this means the following: Let $\left(B_{t}\right)_{t \geq 0}$ be the standard $n$-dimensional Brownian motion. The diffusion equation with generator $L$ as in $(56)$ is the stochastic differential equation:

$$
d Y_{t}=\sqrt{2}\left(\nabla^{2} \psi\left(Y_{t}\right)\right)^{-1 / 2} d B_{t}-\nabla \rho\left(\nabla \psi\left(Y_{t}\right)\right) d t
$$

where $\left(\nabla^{2} \psi(x)\right)^{-1 / 2}$ is the positive-definite square root of $\left(\nabla^{2} \psi(x)\right)^{-1}$. For background on stochastic calculus, the reader may consult sources such as Kallenberg [23] or Øksendal [24]. The stochastic completeness of $M$ is equivalent to the existence of a solution $\left(Y_{t}\right)_{t \geq 0}$ to the equation (60), with an initial condition $Y_{0}=z$ for a fixed $z \in \mathbb{R}^{n}$, that does not explode in finite time. Proposition 4.1 therefore follows from the next proposition:

Proposition 4.5. Let $\psi, V$ and $\rho$ be as in Proposition 4.1 Fix $z \in \mathbb{R}^{n}$. Then there exists a unique stochastic process $\left(Y_{t}\right)_{t \geq 0}$, adapted to the filtration induced by the Brownian motion, such that for all $t \geq 0$,

$$
Y_{t}=z+\int_{0}^{t} \sqrt{2}\left(\nabla^{2} \psi\left(Y_{t}\right)\right)^{-1 / 2} d B_{t}-\int_{0}^{t} \nabla \rho\left(\nabla \psi\left(Y_{t}\right)\right) d t
$$

and such that the map $t \mapsto Y_{t}(t \geq 0)$ is almost-surely continuous.

Proof. Since $\psi(x)$ tends to $+\infty$ when $x \rightarrow \infty$, then the convex set $\{\psi \leq R\}=\{x \in$ $\left.\mathbb{R}^{n} ; \psi(x) \leq R\right\}$ is compact for any $R \in \mathbb{R}$. We use Theorem 21.3 in Kallenberg [23] and the remark following it. We deduce that there exists a unique continuous stochastic process $\left(Y_{t}\right)_{t \geq 0}$ and stopping times $T_{k}=\inf \left\{t \geq 0 ; \psi\left(Y_{t}\right) \geq k\right\}$ such that for any $k>\psi(z), t \geq 0$,

$$
Y_{\min \left\{t, T_{k}\right\}}=z+\int_{0}^{\min \left\{t, T_{k}\right\}} \sqrt{2}\left(\nabla^{2} \psi\left(Y_{t}\right)\right)^{-1 / 2} d B_{t}-\int_{0}^{\min \left\{t, T_{k}\right\}} \nabla \rho\left(\nabla \psi\left(Y_{t}\right)\right) d t .
$$


Denote $T=\sup _{k} T_{k}$. We would like to prove that $T=+\infty$ almost-surely. According to Dynkin's formula and Lemma 4.4, for any $k>\psi(z)$ and $t \geq 0$,

$$
\mathbb{E} \psi\left(Y_{\min \left\{t, T_{k}\right\}}\right)=\psi(z)+\int_{0}^{\min \left\{t, T_{k}\right\}}(L \psi)\left(Y_{t}\right) d t \leq \psi(z)+2 A t,
$$

where $A$ is the parameter from Lemma 4.4. Set $\alpha=-\inf _{x \in \mathbb{R}^{n}} \psi(x)$, a finite number in view of (49). Then $\psi(x)+\alpha$ is non-negative. By Markov-Chebyshev's inequality, for any $t \geq 0$ and $k>\psi(z)$

$$
\mathbb{P}\left(T_{k} \leq t\right)=\mathbb{P}\left(\psi\left(Y_{\min \left\{t, T_{k}\right\}}\right) \geq k\right) \leq \frac{\mathbb{E} \psi\left(Y_{\min \left\{t, T_{k}\right\}}\right)+\alpha}{k+\alpha} \leq \frac{2 A t+\psi(z)+\alpha}{k+\alpha} .
$$

Hence, for any $t \geq 0$,

$$
\mathbb{P}(T \leq t) \leq \inf _{k} \mathbb{P}\left(T_{k} \leq t\right) \leq \liminf _{k \rightarrow \infty} \frac{2 A t+\psi(z)+\alpha}{k+\alpha}=0 .
$$

Therefore $T=+\infty$ almost surely. We may let $k$ tend to infinity in (62) and deduce (61). The uniqueness of the continuous stochastic process $\left(Y_{t}\right)_{t \geq 0}$ that satisfies 61 follows from the uniqueness of the solution to (62).

For $z \in \mathbb{R}^{n}$ write $\left(Y_{t}^{(z)}\right)_{t \geq 0}$ for the stochastic process from Proposition 4.5 with $Y_{0}=z$. Denote by $\nu$ the probability measure on $\mathbb{R}^{n}$ whose density is $e^{-V(x)} d x$. The lemma below is certainly part of the standard theory of diffusion processes. We were not able to find a precise reference, hence we provide a proof which relies on the existence of the heat kernel.

Lemma 4.6. There exists a smooth function $p_{t}(x, y)\left(x, y \in \mathbb{R}^{n}, t>0\right)$ which is symmetric in $x$ and $y$, such that for any $y \in \mathbb{R}^{n}$ and $t>0$, the random vector

$$
Y_{t}^{(y)}
$$

has density $x \mapsto p_{t}(x, y)$ with respect to $\nu$.

Proof. We appeal to Theorem 7.13 and Theorem 7.20 in Grigor'yan [15], which deals with heat kernels on weighted Riemannian manifolds. According to these theorems, there exists a heat kernel, that is, a non-negative function $p_{t}(x, y)\left(x, y \in \mathbb{R}^{n}, t>0\right)$ symmetric in $x$ and $y$ and smooth jointly in $(t, x, y)$, that satisfies the following two properties:

(i) For any $y \in \mathbb{R}^{n}$, the function $u(t, x)=p_{t}(x, y)$ satisfies

$$
\frac{\partial u(t, x)}{\partial t}=L_{x} u(t, x) \quad\left(x \in \mathbb{R}^{n}, t>0\right)
$$

where by $L_{x} u(t, x)$ we mean that the operator $L$ is acting on the $x$-variables.

(ii) For any smooth, compactly-supported function $f: \mathbb{R}^{n} \rightarrow \mathbb{R}$ and $x \in \mathbb{R}^{n}$,

$$
\int_{\mathbb{R}^{n}} p_{t}(x, y) f(y) d \nu(y) \stackrel{t \rightarrow 0^{+}}{\longrightarrow} f(x),
$$

and the convergence in (63) is locally uniform in $x \in \mathbb{R}^{n}$. 
Theorem 7.13 in Grigor'yan [15] also guarantees that $\int p_{t}(x, y) d \nu(x) \leq 1$ for any $y$. It remains to prove that the random vector $Y_{t}^{(y)}$ has density $x \mapsto p_{t}(x, y)$ with respect to $\nu$. Equivalently, we need to show that for any smooth, compactly-supported function $f: \mathbb{R}^{n} \rightarrow$ $\mathbb{R}$ and $y \in \mathbb{R}^{n}, t>0$,

$$
\mathbb{E} f\left(Y_{t}^{(y)}\right)=\int_{\mathbb{R}^{n}} f(x) p_{t}(x, y) d \nu(x) .
$$

Denote by $v(t, y) \quad\left(t>0, y \in \mathbb{R}^{n}\right)$ the right-hand side of (64), a smooth, bounded function. We also set $v(0, y)=f(y) \quad\left(y \in \mathbb{R}^{n}\right)$ by continuity, according to (ii). Then the function $v(t, y)$ is continuous and bounded in $(t, y) \in[0,+\infty) \times \mathbb{R}^{n}$. Since $f$ is compactly-supported then we may safely differentiate under the integral sign with respect to $y$ and $t$, and obtain

$$
\frac{\partial v(t, y)}{\partial t}=\int_{\mathbb{R}^{n}} f(x) \frac{\partial p_{t}(x, y)}{\partial t} d \nu(y), \quad L_{y} v(t, y)=\int_{\mathbb{R}^{n}} f(x)\left(L_{y} p_{t}(x, y)\right) d \nu(y) .
$$

From (i) we learn that

$$
\frac{\partial v(t, y)}{\partial t}=L_{y} v(t, y) \quad\left(y \in \mathbb{R}^{n}, t>0\right) .
$$

Fix $t_{0}>0$ and $y \in \mathbb{R}^{n}$. Denote $Z_{t}=v\left(t_{0}-t, Y_{t}^{(y)}\right)$ for $0 \leq t \leq t_{0}$. Then $\left(Z_{t}\right)_{0 \leq t \leq t_{0}}$ is a continuous stochastic process. From Itô's formula and (65), for $0 \leq t \leq t_{0}$,

$$
Z_{t}=Z_{0}+R_{t}+\int_{0}^{t}\left[L_{y} v\left(t_{0}-t, Y_{t}^{(y)}\right)-\frac{\partial v}{\partial t}\left(t_{0}-t, Y_{t}^{(y)}\right)\right] d t=Z_{0}+R_{t}
$$

where $\left(R_{t}\right)_{0 \leq t \leq t_{0}}$ is a local martingale with $R_{0}=0$. Since $v$ is bounded, then $\left(R_{t}\right)_{0 \leq t \leq t_{0}}$ is in fact a martingale, and in particular $\mathbb{E} R_{t_{0}}=\mathbb{E} R_{0}=0$. Thus,

$$
\mathbb{E} f\left(Y_{t_{0}}^{(y)}\right)=\mathbb{E} Z_{t_{0}}=\mathbb{E} Z_{0}=v\left(t_{0}, y\right)=\int_{\mathbb{R}^{n}} f(x) p_{t_{0}}(x, y) d \nu(x),
$$

and (64) is proven.

Corollary 4.7. Suppose that $Z$ is a random vector in $\mathbb{R}^{n}$, distributed according to $\nu$, independent of the Brownian motion $\left(B_{t}\right)_{t \geq 0}$ used for the construction of $\left(Y_{t}^{(z)}\right)_{t \geq 0, z \in \mathbb{R}^{n}}$.

Then, for any $t \geq 0$, the random vector $Y_{t}^{(Z)}$ is also distributed according to $\nu$.

Proof. According to Lemma4.6, for any measurable set $A \subset \mathbb{R}^{n}$,

$$
\begin{aligned}
\mathbb{P}\left(Y_{t}^{(Z)} \in A\right) & =\int_{\mathbb{R}^{n}} \mathbb{P}\left(Y_{t}^{(z)} \in A\right) d \nu(z)=\int_{\mathbb{R}^{n}}\left(\int_{A} p_{t}(z, x) d \nu(x)\right) d \nu(z) \\
& =\int_{A}\left(\int_{\mathbb{R}^{n}} p_{t}(x, z) d \nu(z)\right) d \nu(x)=\nu(A) .
\end{aligned}
$$

Remark 4.8. Our choice to use stochastic processes in this paper is just a matter of personal taste. All of the arguments here can be easily rephrased in analytic terminology. For instance, the proof of Proposition 4.5 relies on the fact that $L \psi$ is bounded from above, similarly to the analytic approach in Grigor'yan [15, Section 8.4]. Another example is the use of local martingales towards the end of Lemma 4.6, which may be replaced by analytic arguments as in [15, Section 7.4]. 


\section{Bakry-Émery technique}

In this section we prove Theorem 1.1. While the viewpoint and ideas of Bakry and Émery [4] are certainly the main source of inspiration for our analysis, we are not sure whether the abstract framework in [3, 4] entirely encompasses the subtlety of our specific weighted Riemannian manifold. For instance, Lemma 5.2 below seems related to the positivity of the carré du champ $\Gamma_{2}$ and to the inequality $\Gamma_{2} \geq \Gamma / 2$, rendered as property (ii) in Section 1 above. In the case $\varepsilon \geq 1 / 2$, Lemma 5.2] actually follows from an application of [3, Lemma 2.4] with $f(x)=x^{1}$ and $\rho=1 / 2$. Yet, in general, it appears to us advantageous to proceed by analyzing our model for itself, rather than viewing it as an abstract diffusion semigroup satisfying a curvature-dimension bound.

Let $\mu$ be a log-concave probability measure on $\mathbb{R}^{n}$ satisfying the regularity assumptions (1), whose barycenter lies at the origin. Let $\psi: \mathbb{R}^{n} \rightarrow \mathbb{R}$ be convex and smooth, such that the transport equation (3) holds true. In Section 4 we proved that $M_{\mu}^{*}$ is stochastically complete. Since $M_{\mu^{*}}$ is isomorphic to $M_{\mu}$, then $M_{\mu}$ is also stochastically complete.

Let us describe in greater detail the diffusion process associated with $M_{\mu}=\left(K, \nabla^{2} \varphi, \mu\right)$. Recall that the Legendre transform $\varphi=\psi^{*}$ is smooth and convex on $K$, and that

$$
\varphi(x)+\psi(\nabla \varphi(x))=x \cdot \nabla \varphi(x) \quad(x \in K) .
$$

We may rephrase (3) in terms of $\varphi=\psi^{*}$, and using $\left(\nabla^{2} \varphi(x)\right)^{-1}=\nabla^{2} \psi(\nabla \varphi(x))$, we arrive at the equation

$$
\operatorname{det} \nabla^{2} \varphi(x)=e^{x \cdot \nabla \varphi(x)-\varphi(x)-\rho(x)} \quad(x \in K) .
$$

The Hessian matrix $\nabla^{2} \varphi$ is invertible everywhere, so we write $\left(\nabla^{2} \varphi(x)\right)^{-1}=\left(\varphi^{i j}(x)\right)_{i, j=1, \ldots, n}$, and as before we also use abbreviations such as $\varphi_{i}^{j k}=\varphi^{j \ell} \varphi^{k m} \varphi_{i \ell m}$. In this section, for a smooth function $u: K \rightarrow \mathbb{R}$, denote

$$
L u(x)=\varphi^{i j} u_{i j}-x^{i} u_{i} \quad \text { for } x=\left(x^{1}, \ldots, x^{n}\right) \in K .
$$

Lemma 5.1. The operator L from (67) is the Laplacian associated with the weighted Riemannian manifold $M_{\mu}$.

Proof. By taking the logarithmic derivative of (66) and arguing as in the proof of Lemma 4.3, we obtain that for any $x \in K, i=1, \ldots, n$,

$$
\varphi_{j}^{i j}=x^{i}-\varphi^{i j} \rho_{j} .
$$

Integrating by parts and using (68), we see that for any two smooth functions $u, v: K \rightarrow \mathbb{R}$ with one of them compactly-supported,

$$
\int_{K} \varphi^{i j} u_{i} v_{j} d \mu=-\int_{K} v\left(\varphi^{i j} u_{i j}-\left(\varphi_{j}^{i j}+\varphi^{i j} \rho_{j}\right) u_{i}\right) e^{-\rho}=-\int_{K} v(L u) d \mu .
$$

Lemma 5.2. Fix $\varepsilon>0$. For $x \in K$ set $f(x)=\varphi^{11}(x)$. Then,

$$
L\left(f^{\varepsilon}\right)+\varepsilon f^{\varepsilon} \geq 0 .
$$


Proof. For $i, j=1, \ldots, n$,

$$
f_{i}=\left(\varphi^{11}\right)_{i}=-\varphi^{1 k} \varphi^{1 \ell} \varphi_{i k \ell}, \quad f_{i j}=-\varphi_{i j}^{11}+2 \varphi_{j}^{1 k} \varphi_{i k}^{1}
$$

Therefore,

$$
L f=\varphi^{i j} f_{i j}-x^{i} f_{i}=-\varphi_{j}^{11 j}+2 \varphi_{i}^{1 j} \varphi_{j}^{1 i}+x^{j} \varphi_{j}^{11} .
$$

Taking the logarithm of (66) and differentiating with respect to $x^{i}$ and $x^{\ell}$, we see that

$$
\varphi_{j i \ell}^{j}-\varphi_{i}^{j k} \varphi_{j k \ell}=-\rho_{i \ell}+\varphi_{i \ell}+x^{j} \varphi_{i \ell j} \quad(i, \ell=1, \ldots, n) .
$$

Multiplying by $\varphi^{1 i} \varphi^{1 \ell}$ and summing yields

$$
\varphi_{j}^{j 11}-\varphi_{k}^{1 j} \varphi_{j}^{1 k}=-\varphi^{1 i} \varphi^{1 \ell} \rho_{i \ell}+\varphi^{11}+x^{j} \varphi_{j}^{11} .
$$

Since $\rho$ is convex then its Hessian matrix is non-negative definite and $\rho_{i \ell} \varphi^{1 i} \varphi^{1 \ell} \geq 0$. From (69) and (70),

$$
L f=\varphi_{k}^{1 j} \varphi_{j}^{1 k}-\varphi^{11}+\rho_{i \ell} \varphi^{1 i} \varphi^{1 \ell} \geq \varphi_{k}^{1 j} \varphi_{j}^{1 k}-\varphi^{11}=\varphi_{k}^{1 j} \varphi_{j}^{1 k}-f .
$$

The chain rule of the Laplacian is $L(\lambda(f))=\lambda^{\prime}(f) L f+\lambda^{\prime \prime}(f) \varphi^{i j} f_{j} f_{j}$, as may be verified directly. Using the chain rule with $\lambda(t)=t^{\varepsilon}$ we see that (71) leads to

$$
L\left(f^{\varepsilon}\right)=\varepsilon f^{\varepsilon-1} L f+\varepsilon(\varepsilon-1) f^{\varepsilon-2} \varphi^{11 j} \varphi_{j}^{11} \geq \varepsilon f^{\varepsilon-1} \varphi_{k}^{1 j} \varphi_{j}^{1 k}-\varepsilon f^{\varepsilon}+\varepsilon(\varepsilon-1) f^{\varepsilon-2} \varphi^{11 j} \varphi_{j}^{11} .
$$

That is,

$$
L\left(f^{\varepsilon}\right)+\varepsilon f^{\varepsilon} \geq \varepsilon f^{\varepsilon-1}\left[\varphi_{k}^{1 j} \varphi_{j}^{1 k}+(\varepsilon-1) \frac{\varphi^{11 j} \varphi_{j}^{11}}{\varphi^{11}}\right] \geq \varepsilon f^{\varepsilon-1}\left[\varphi_{k}^{1 j} \varphi_{j}^{1 k}-\frac{\varphi^{11 j} \varphi_{j}^{11}}{\varphi^{11}}\right]
$$

where we used the fact that $\varphi^{11 j} \varphi_{j}^{11} \geq 0$ in the last passage (or more generally, $\varphi^{i j} h_{i} h_{j} \geq 0$ for any smooth function $h$ ). It remains to show that the right-hand side of (72) is nonnegative. Denote $A=\left(\varphi_{k}^{1 j}\right)_{j, k=1, \ldots, n}$. The matrix $B=\left(\varphi^{1 j k}\right)_{j, k=1, \ldots, n}$ is a symmetric matrix, since $\varphi^{1 j k}=\varphi^{1 \ell} \varphi^{j m} \varphi^{k r} \varphi_{\ell m r}$. We have $A=\left(\nabla^{2} \varphi\right) B$, and hence

$$
\varphi_{k}^{1 j} \varphi_{j}^{1 k}=\operatorname{Tr}\left(A^{2}\right)=\operatorname{Tr}\left[\left(\left(\nabla^{2} \varphi\right)^{1 / 2} B\left(\nabla^{2} \varphi\right)^{1 / 2}\right)^{2}\right]=\left\|\left(\nabla^{2} \varphi\right)^{1 / 2} B\left(\nabla^{2} \varphi\right)^{1 / 2}\right\|_{H S}^{2}
$$

since the matrix $\left(\nabla^{2} \varphi\right)^{1 / 2} B\left(\nabla^{2} \varphi\right)^{1 / 2}$ is symmetric, where $\|T\|_{H S}$ stands for the HilbertSchmidt norm of the matrix $T$. We will use the fact that the Hilbert-Schmidt norm is at least as large as the operator norm, that is, $\|T\|_{H S}^{2} \geq|T x|^{2} /|x|^{2}$ for any $0 \neq x \in \mathbb{R}^{n}$. Setting $e_{1}=(1,0, \ldots, 0)$, we conclude that

$$
\varphi_{k}^{1 j} \varphi_{j}^{1 k} \geq \frac{\left|\left(\nabla^{2} \varphi\right)^{1 / 2} B\left(\nabla^{2} \varphi\right)^{1 / 2}\left(\nabla^{2} \varphi\right)^{-1 / 2} e_{1}\right|^{2}}{\left|\left(\nabla^{2} \varphi\right)^{-1 / 2} e_{1}\right|^{2}}=\frac{\varphi^{11 i} \varphi_{i j} \varphi^{11 j}}{\varphi^{11}}=\frac{\varphi_{j}^{11} \varphi^{11 j}}{\varphi^{11}} .
$$

The lemma follows from (72) and (73). 
Let $\left(B_{t}\right)_{t \geq 0}$ be the standard $n$-dimensional Brownian motion. From the results of Section 4 the diffusion process whose generator is $L$ from (67) is well-defined. That is, there exists a unique stochastic process $\left(X_{t}^{(z)}\right)_{t \geq 0, z \in K}$, continuous in $t$ and adapted to the filtration induced by the Brownian motion, such that for all $t \geq 0$,

$$
X_{t}^{(z)}=z+\int_{0}^{t} \sqrt{2}\left(\nabla^{2} \varphi\left(X_{t}^{(z)}\right)\right)^{-1 / 2} d B_{t}-\int_{0}^{t} X_{t}^{(z)} d t .
$$

Our proof of Theorem 1.1 relies on a few lemmas in which the main technical obstacle is to prove the integrability of certain local martingales.

Lemma 5.3. Fix $z \in K$ and set $X_{t}=X_{t}^{(z)}(t \geq 0)$. Then for any $t \geq 0$,

$$
\mathbb{E} X_{t}=e^{-t} z
$$

and for any $\theta \in S^{n-1}$,

$$
e^{2 t} \mathbb{E}\left(X_{t} \cdot \theta\right)^{2} \geq(z \cdot \theta)^{2}+2 \int_{0}^{t} e^{2 s} \mathbb{E}\left[\left(\nabla^{2} \varphi\right)^{-1}\left(X_{s}\right) \theta \cdot \theta\right] d s .
$$

Proof. From Itô's formula and (74),

$$
d\left(e^{t} X_{t}\right)=e^{t} d X_{t}+e^{t} X_{t} d t=\sqrt{2} e^{t}\left(\nabla^{2} \varphi\left(X_{t}\right)\right)^{-1 / 2} d B_{t} .
$$

Therefore $\left(e^{t} X_{t}\right)_{0 \leq t \leq T}$ is a local martingale, for any fixed number $T>0$. However, $e^{t} X_{t} \in$ $e^{T} K$ for $0 \leq t \leq T$, and $K \subset \mathbb{R}^{n}$ is a bounded set. Therefore $\left(e^{t} X_{t}\right)_{0 \leq t \leq T}$ is a bounded process, and hence it is a martingale. We conclude that

$$
\mathbb{E} e^{t} X_{t}=\mathbb{E} e^{0} X_{0}=z \quad(t \geq 0),
$$

and (75) is proven. It remains to prove (76). Without loss of generality we may assume that $\theta=e_{1}=(1,0, \ldots, 0)$. Denote $Z_{t}=e^{2 t}\left(X_{t} \cdot e_{1}\right)^{2}$. According to (74) and Itô's formula, for any $t \geq 0$,

$$
Z_{t}=\left(z \cdot e_{1}\right)^{2}+M_{t}+\int_{0}^{t}\left(2 e^{2 s} \varphi^{11}\left(X_{s}\right)\right) d s
$$

where $\left(M_{t}\right)_{t \geq 0}$ is a local martingale with $M_{0}=0$. Since $\varphi^{11}$ is positive, then for any $t \geq 0$,

$$
Z_{t}-\left(z \cdot e_{1}\right)^{2} \geq M_{t}
$$

Since $K$ is bounded, then $\left(Z_{t}\right)_{0 \leq t \leq T}$ is a bounded process, for any number $T>0$. According to (78), the local martingale $\left(M_{t}\right)_{0 \leq t \leq T}$ is bounded from above, and by Fatou's lemma it is a sub-martingale. In particular $\mathbb{E} M_{t} \geq \mathbb{E} M_{0}=0$ for any $t$. From (77),

$$
\mathbb{E} Z_{t} \geq\left(z \cdot e_{1}\right)^{2}+2 \mathbb{E} \int_{0}^{t} e^{2 s} \varphi^{11}\left(X_{s}\right) d s \quad(t \geq 0) .
$$

Since $\mathbb{E} Z_{t}<+\infty$ and $\varphi^{11}$ is positive, we may use Fubini's theorem to conclude that for any $t \geq 0$

$$
\mathbb{E} Z_{t} \geq\left(z \cdot e_{1}\right)^{2}+2 \int_{0}^{t} e^{2 s} \mathbb{E} \varphi^{11}\left(X_{s}\right) d s
$$


Remark 5.4. Once Theorem 1.1 is established, we can prove that equality holds in (76). Indeed, it follows from Theorem 1.1 and (77) that $\left(M_{t}\right)_{0 \leq t \leq T}$ is a bounded process and hence a martingale.

Lemma 5.5. Assume that the convex body $K$ has a smooth boundary and that its Gauss curvature is positive everywhere. Assume also that there exists $\varepsilon_{0}>0$ with

$$
\nabla^{2} \rho(x) \geq \varepsilon_{0} \cdot I d \quad(x \in K)
$$

in the sense of symmetric matrices. Fix $z \in K$ and set $X_{t}=X_{t}^{(z)}(t \geq 0)$. Denote $f(x)=\varphi^{11}(x)$ for $x \in K$. Then, for any $t, \varepsilon>0$,

$$
f(z) \leq e^{t}\left(\mathbb{E} f^{\varepsilon}\left(X_{t}\right)\right)^{1 / \varepsilon} .
$$

Proof. Our assumptions enable the application of Proposition 3.1. According to the conclusion of Proposition 3.1 there exists $M>0$ such that

$$
\nabla^{2} \psi(y) \leq M \cdot I d \quad\left(y \in \mathbb{R}^{n}\right) .
$$

Since $\left(\nabla^{2} \varphi\right)^{-1}(x)=\nabla^{2} \psi(\nabla \varphi(x))$, then,

$$
f(x)=\varphi^{11}(x) \leq M \quad(x \in K) .
$$

From Itô's formula and (74),

$$
e^{\varepsilon t} f^{\varepsilon}\left(X_{t}\right)=f^{\varepsilon}(z)+M_{t}+\int_{0}^{t} e^{\varepsilon s}\left[\left(L f^{\varepsilon}\right)\left(X_{s}\right)+\varepsilon f^{\varepsilon}\left(X_{s}\right)\right] d s
$$

where $M_{t}$ is a local martingale with $M_{0}=0$. According to (82) and Lemma 5.2 for any $t \geq 0$,

$$
e^{\varepsilon t} f^{\varepsilon}\left(X_{t}\right) \geq f^{\varepsilon}(z)+M_{t} .
$$

We may now use (81) and (83) in order to conclude that the local martingale $\left(M_{t}\right)_{0 \leq t \leq T}$ is bounded from above, for any number $T>0$. Hence it is a sub-martingale, and $\mathbb{E} M_{t} \geq$ $\mathbb{E} M_{0}=0$ for any $t \geq 0$. Now (80) follows by taking the expectation of 83].

Remark 5.6. We will only use (80) for $\varepsilon=1$, even though the statement for a small $\varepsilon$ is much stronger. In the limit where $\varepsilon$ tends to zero, it is not too difficult to prove that the right-hand side of (80) approaches $\exp \left(t+\mathbb{E} \log f\left(X_{t}\right)\right)$.

The covariance matrix of a square-integrable random vector $Z=\left(Z_{1}, \ldots, Z_{n}\right) \in \mathbb{R}^{n}$ is defined to be

$$
\operatorname{Cov}(Z)=\left(\mathbb{E} Z_{i} Z_{j}-\mathbb{E} Z_{i} \cdot \mathbb{E} Z_{j}\right)_{i, j=1, \ldots, n}
$$


Corollary 5.7. Assume that the convex body $K$ has a smooth boundary and that its Gauss curvature is positive everywhere. Assume also that there exists $\varepsilon_{0}>0$ with

$$
\nabla^{2} \rho(x) \geq \varepsilon_{0} \cdot I d \quad(x \in K) .
$$

Then for any $z \in K$ and $t>0$,

$$
\left(\nabla^{2} \varphi\right)^{-1}(z) \leq \frac{e^{2 t}}{2\left(e^{t}-1\right)} \cdot \operatorname{Cov}\left(X_{t}^{(z)}\right)
$$

in the sense of symmetric matrices.

Proof. Fix $z \in K, t>0$ and $\theta \in S^{n-1}$. We need to prove that

$$
\left(\nabla^{2} \varphi(z)\right)^{-1} \theta \cdot \theta \leq \frac{e^{2 t}}{2\left(e^{t}-1\right)} \operatorname{Var}\left(X_{t}^{(z)} \cdot \theta\right) .
$$

Without loss of generality we may assume that $\theta=e_{1}=(1,0, \ldots, 0)$. We use Lemma 5.3 and also Lemma 5.5 with $\varepsilon=1$, and obtain

$$
e^{2 t} \mathbb{E}\left(X_{t}^{(z)} \cdot e_{1}\right)^{2} \geq\left(z \cdot e_{1}\right)^{2}+2 \int_{0}^{t} e^{2 s} \mathbb{E} \varphi^{11}\left(X_{s}^{(z)}\right) d s \geq\left(z \cdot e_{1}\right)^{2}+2 \varphi^{11}(z) \int_{0}^{t} e^{s} d s .
$$

Recall that $\mathbb{E} X_{t}^{(z)}=e^{-t} z$, according to Lemma 5.3. Consequently,

$$
\varphi^{11}(z) \leq \frac{e^{2 t}}{2\left(e^{t}-1\right)}\left(\mathbb{E}\left(X_{t}^{(z)} \cdot e_{1}\right)^{2}-\left(e^{-t} z \cdot e_{1}\right)^{2}\right)=\frac{e^{2 t}}{2\left(e^{t}-1\right)} \operatorname{Var}\left(X_{t}^{(z)} \cdot e_{1}\right),
$$

and (85) is proven for $\theta=e_{1}$.

Proof of Theorem 1.1 Assume first that the convex body $K$ has a smooth boundary, that its Gauss curvature is positive everywhere, and that there exists $\varepsilon_{0}$ for which (84) holds true. We apply Corollary 5.7 with $t=\log 2$, and conclude that for any $z \in K$,

$$
\operatorname{Tr}\left[\left(\nabla^{2} \varphi\right)^{-1}(z)\right] \leq 2 \operatorname{Tr}\left[\operatorname{Cov}\left(X_{t}^{(z)}\right)\right] \leq 2 \mathbb{E}\left|X_{t}^{(z)}\right|^{2} \leq 2 R^{2}(K)
$$

as $X_{t}^{(z)} \in K$ almost surely. Therefore, for any $x \in \mathbb{R}^{n}$, setting $z=\nabla \psi(x)$ we have

$$
\Delta \psi(x)=\operatorname{Tr}\left[\nabla^{2} \psi(x)\right]=\operatorname{Tr}\left[\left(\nabla^{2} \varphi\right)^{-1}(z)\right] \leq 2 R^{2}(K) .
$$

It still remains to eliminate the extra strict-convexity assumptions. To that end, we select a sequence of smooth convex bodies $K_{\ell} \subset \mathbb{R}^{n}$, each with a positive Gauss curvature, that converge in the Hausdorff metric to $K$. We then consider a sequence of log-concave probability measures $\mu_{\ell}$ with barycenter at the origin that converge weakly to $\mu$, such that $\mu_{\ell}$ is supported on $K_{\ell}$ and such that the smooth density of $\mu_{\ell}$ satisfies (84) with, say, $\varepsilon_{0}=1 / \ell$. We also assume that $\mu_{\ell}$ and $K_{\ell}$ satisfy the regularity conditions (1).

It is not very difficult to construct the $\mu_{\ell}$ 's: For instance, convolve $\mu$ with a tiny Gaussian (this preserves log-concavity), multiply the density by $\exp \left(-|x|^{2} / \ell\right)$, truncate with $K_{\ell}$ and 
translate a little so that the barycenter would lie at the origin. This way we obtain a sequence of smooth, convex functions $\psi_{\ell}: \mathbb{R}^{n} \rightarrow \mathbb{R}$ such that $\mu_{\ell}$ is the moment measure of $\psi_{\ell}$. We may translate, and assume that $\psi$ and each of the $\psi_{\ell}^{\prime} s$ are centered, in the terminology of Section 2 According to (86), we know that

$$
\Delta \psi_{\ell}(x) \leq 2 R^{2}\left(K_{\ell}\right) \quad\left(x \in \mathbb{R}^{n}, \ell \geq 1\right) .
$$

Furthermore, $\mu_{\ell} \longrightarrow \mu$ weakly, and by Proposition 2.1, also $\psi_{\ell} \longrightarrow \psi$ pointwise in $\mathbb{R}^{n}$. Since $\psi_{\ell}$ and $\psi$ are smooth, then [26, Theorem 24.5] implies that

$$
\nabla \psi_{\ell}(x) \stackrel{\ell \rightarrow \infty}{\longrightarrow} \nabla \psi(x) \quad\left(x \in \mathbb{R}^{n}\right) .
$$

The function $\psi_{\ell}$ is $R\left(K_{\ell}\right)$-Lipschitz, and $R\left(K_{\ell}\right) \longrightarrow R(K)$. Hence $\sup _{\ell, x}\left|\nabla \psi_{\ell}(x)\right|$ is finite. By the bounded convergence theorem, for any $x_{0} \in \mathbb{R}^{n}$ and $\varepsilon>0$,

$$
\int_{B\left(x_{0}, \varepsilon\right)} \Delta \psi_{\ell}=\int_{\partial B\left(x_{0}, \varepsilon\right)} \nabla \psi_{\ell} \cdot N \stackrel{\ell \rightarrow \infty}{\longrightarrow} \int_{\partial B\left(x_{0}, \varepsilon\right)} \nabla \psi \cdot N=\int_{B\left(x_{0}, \varepsilon\right)} \Delta \psi,
$$

where $N$ is the outer unit normal. From (87) and (88) we conclude that for any $x_{0} \in \mathbb{R}^{n}$ and $\varepsilon>0$,

$$
\int_{B\left(x_{0}, \varepsilon\right)} \Delta \psi \leq \operatorname{Vol}_{n}\left(B\left(x_{0}, \varepsilon\right)\right) \cdot \limsup _{\ell \rightarrow \infty} 2 R^{2}\left(K_{\ell}\right)=2 \operatorname{Vol}_{n}\left(B\left(x_{0}, \varepsilon\right)\right) R^{2}(K),
$$

where $\operatorname{Vol}_{n}$ is the Lebesgue measure in $\mathbb{R}^{n}$. Since $\psi$ is smooth, then we may let $\varepsilon$ tend to zero and conclude that $\Delta \psi\left(x_{0}\right) \leq 2 R^{2}(K)$, for any $x_{0} \in \mathbb{R}^{n}$.

Posteriori, we may strengthen Corollary 5.7 and eliminate the strict-convexity assumptions. These assumptions were used only in the proof of Lemma 5.5, to deduce the existence of some number $M>0$ for which $\nabla^{2} \psi(x) \leq M \cdot I d$, for all $x \in \mathbb{R}^{n}$. Theorem 1.1 provides such a number $M=2 R^{2}(K)$, without any strict-convexity assumptions on $\rho$ or $K$. We may therefore upgrade Corollary 5.7, and conclude that

Corollary 5.8. Whenever $\mu$ is a log-concave probability measure with barycenter at the origin, satisfying the regularity conditions (1), we have

$$
\left(\nabla^{2} \varphi\right)^{-1}(z) \leq \frac{e^{2 t}}{2\left(e^{t}-1\right)} \cdot \operatorname{Cov}\left(X_{t}^{(z)}\right)
$$

in the sense of symmetric matrices, for any $z \in K$ and $t>0$.

\section{The Brascamp-Lieb inequality as a Poincaré inequal- ity}

We retain the assumptions and notation of the previous section. That is, $\mu$ is a log-concave probability measure on $\mathbb{R}^{n}$, with barycenter at the origin, that satisfies the regularity assumptions (1). The measure $\mu$ is the moment-measure of the smooth and convex function 
$\psi: \mathbb{R}^{n} \rightarrow \mathbb{R}$. Equation (3) holds true, and we denote $\varphi=\psi^{*}$. According to the BrascampLieb inequality [8], for any smooth function $u: \mathbb{R}^{n} \rightarrow \mathbb{R}$ such that $u e^{-\psi}$ is integrable,

$$
\int_{\mathbb{R}^{n}} u e^{-\psi}=0 \quad \Longrightarrow \quad \int_{\mathbb{R}^{n}} u^{2} e^{-\psi} \leq \int_{\mathbb{R}^{n}}\left[\left(\nabla^{2} \psi\right)^{-1} \nabla u \cdot \nabla u\right] e^{-\psi}
$$

Equality in (89) holds when $u(x)=\nabla \psi(x) \cdot \theta$ for some $\theta \in \mathbb{R}^{n}$. Note that (89) is precisely the Poincare inequality with the best constant of the weighted Riemannian manifold $M_{\mu}^{*}$. By using the isomorphism between $M_{\mu}$ and $M_{\mu}^{*}$, we translate (89) as follows: For any smooth function $f: K \rightarrow \mathbb{R}$ which is $\mu$-integrable,

$$
\operatorname{Var}_{\mu}(f) \leq \int_{K}\left(\varphi^{i j} f_{i} f_{j}\right) d \mu
$$

where $\operatorname{Var}_{\mu}(f)=\int f^{2} d \mu-\left(\int f d \mu\right)^{2}$. Equality in (90) holds when $f(x)=A+x \cdot \theta$ for some $\theta \in \mathbb{R}^{n}$ and $A \in \mathbb{R}$. This is in accordance with the fact that linear functions are eigenfunctions, i.e.,

$$
L x^{i}=-x^{i} \quad(i=1, \ldots, n)
$$

where $L u=\varphi^{i j} u_{i j}-x^{i} u_{i}$ is the Laplacian of the weighted Riemannian manifold $M_{\mu}$. In fact, (90) means that the spectrum of the (Friedrich extension of the) operator $L$ cannot intersect the interval $(-1,0)$, and that the restriction of $-L$ to the subspace of mean-zero functions is at least the identity operator, in the sense of symmetric operators.

Theorem 1.1 states that $\Delta \Psi(x) \leq 2 R^{2}(K)$ everywhere in $\mathbb{R}^{n}$. A weak conclusion is that $\nabla^{2} \psi(x) \leq 2 R^{2}(K) \cdot I d$, or rather, that $\left(\nabla^{2} \varphi(x)\right)^{-1} \leq 2 R^{2}(K) \cdot I d$. By substituting this information into (90), we see that for any smooth function $f \in L^{1}(\mu)$,

$$
\operatorname{Var}_{\mu}(f) \leq 2 R^{2}(K) \int_{K}|\nabla f|^{2} d \mu .
$$

This completes the proof of Corollary 1.2 See [19] for more Poincaré-type inequalities that are obtained by imposing a Riemannian structure on the convex body $K$. The KannanLovasź-Simonovits conjecture speculates that $R^{2}(K)$ in (91) may be replaced by a universal constant times $\|\operatorname{Cov}(\mu)\|$, where $\operatorname{Cov}(\mu)$ is the covariance matrix of the random vector that is distributed according to $\mu$, and $\|\cdot\|$ is the operator norm.

A potential way to make progress towards the Kannan-Lovasź-Simonovits conjecture is to try to bound the matrices $\left(\nabla^{2} \varphi\right)^{-1}(x)(x \in K)$ in terms of $\operatorname{Cov}(\mu)$. The following proposition provides a modest step in this direction:

Proposition 6.1. Fix $\theta \in S^{n-1}$ and denote

$$
V=\int_{\mathbb{R}^{n}}(x \cdot \theta)^{2} d \mu(x) .
$$

Then, for any $p \geq 1$,

$$
\left(\int_{K}\left|\frac{\left(\nabla^{2} \varphi\right)^{-1} \theta \cdot \theta}{V}\right|^{p} d \mu\right)^{1 / p} \leq 4 p^{2} .
$$


Proof. Without loss of generality, assume that $\theta=e_{1}=(1,0, \ldots, 0)$. According to Corollary 5.8 for any $z \in K$ and $t>0$,

$$
\varphi^{11}(z) \leq \frac{e^{2 t}}{2\left(e^{t}-1\right)} \operatorname{Var}\left(X_{t}^{(z)} \cdot e_{1}\right) \leq \frac{e^{2 t}}{2\left(e^{t}-1\right)} \mathbb{E}\left(X_{t}^{(z)} \cdot e_{1}\right)^{2} .
$$

Let $Z$ be a random vector that is distributed according to $\mu$, independent of the Brownian motion used in the construction of the process $\left(X_{t}^{(z)}\right)_{t \geq 0, z \in K}$. It follows from Corollary 4.7 that for any fixed $t \geq 0$ the random vector $X_{t}^{(Z)}$ is also distributed according to $\mu$. By setting $t=\log 2$ in (92) and applying Hölder's inequality, we see that for any $p \geq 1$,

$$
\mathbb{E}\left|\varphi^{11}(Z)\right|^{p} \leq 2^{p} \mathbb{E}\left|X_{t}^{(Z)} \cdot e_{1}\right|^{2 p}=2^{p} \mathbb{E}\left|Z \cdot e_{1}\right|^{2 p} .
$$

The random vector $Z$ has a log-concave density. According to the Berwald inequality [6, 7],

$$
\left(\mathbb{E}\left|Z \cdot e_{1}\right|^{2 p}\right)^{1 /(2 p)} \leq \frac{\Gamma(2 p+1)^{1 /(2 p)}}{\Gamma(3)^{1 / 2}} \sqrt{\mathbb{E}\left|Z \cdot e_{1}\right|^{2}} \leq \frac{2 p}{\sqrt{2}} \sqrt{V}
$$

(The Berwald inequality is formulated in [6, 7] for the uniform measure on a convex body, but it is well-known that is applies for all log-concave probability measures. For instance, one may deduce the log-concave version from the convex-body version by using a marginal argument as in [17]). The proposition follows from (93) and (94).

There are several heuristic arguments that indicate much better bounds for $\left(\nabla^{2} \varphi\right)^{-1}$ than the ones proven in this paper. These better bounds, in turn, could lead to interesting Poincaré-type inequalities for log-concave measures. At the moment, it is not clear to us how to rigorously justify these heuristic arguments, partly because of the annoying fact that our Riemannian structure on $K$ is not geodesically complete. Still, hoping that this work will be continued, we decided to add the Roman numeral I at the end of its title.

\section{References}

[1] Abreu, M., Kähler geometry of toric manifolds in symplectic coordinates. Symplectic and contact topology: interactions and perspectives, Fields Inst. Commun., Vol. 35, Amer. Math. Soc., (2003), 1-24.

[2] Alesker, S., Dar, S., Milman, V., A remarkable measure preserving diffeomorphism between two convex bodies in $\mathbb{R}^{n}$. Geom. Dedicata, Vol. 74, No. 2, (1999), 201-212.

[3] Bakry, D., On Sobolev and logarithmic Sobolev inequalities for Markov semigroups. New trends in stochastic analysis (Charingworth, 1994), World Sci. Publ., River Edge, NJ, (1997), 43-75.

[4] Bakry, D., Émery, M., Diffusions hypercontractives. Séminaire de probabilités, XIX, 1983/84, Springer Lecture Notes in Math., Vol. 1123, (1985), 177-206.

[5] Berman, R. J., Berndtsson, B., Real Monge-Ampère equations and Kähler-Ricci solitons on toric log Fano varieties. Preprint, arXiv:1207.6128 
[6] Berwald. L., Verallgemeinerung eines Mittelwertsatzes von J. Favard, für positive konkave Funktionen. Acta Math., Vol. 79, (1947), 17-37.

[7] Borell, C., Complements of Lyapunov's inequality. Math. Ann., Vol. 205, (1973), 323331.

[8] Brascamp, H. J., Lieb, E. H., On extensions of the Brunn-Minkowski and PrékopaLeindler theorems, including inequalities for log concave functions, and with an application to the diffusion equation. J. Funct. Anal., Vol. 22, No. 4, (1976), 366-389.

[9] Caffarelli, L., The regularity of mappings with a convex potential. J. Amer. Math. Soc., Vol. 5, (1992), 99-104.

[10] Caffarelli, L., Monotonicity properties of optimal transportation and the FKG and related inequalities. Comm. Math. Phys., Vol. 214, No. 3, (2000), 547-563.

[11] Caffarelli, L., Erratum: "Monotonicity of optimal transportation and the FKG and related inequalities". Comm. Math. Phys., Vol. 225, No. 2, (2002), 449-450.

[12] Cordero-Erausquin, D., Klartag, B., Moment measures. Preprint, arXiv:1304.0630

[13] Donaldson, S. K., Kähler geometry on toric manifolds, and some other manifolds with large symmetry. Handbook of geometric analysis. Adv. Lect. Math. (ALM), Vol. 7, No. 1, Int. Press, Somerville, MA, (2008), 29-75.

[14] Fradelizi, M., Sections of convex bodies through their centroid. Arch. Math., Vol. 69, No. 6, (1997), 515-522.

[15] Grigor'yan, A., Heat kernel and analysis on manifolds. AMS/IP Studies in Advanced Mathematics, Vol. 47, Amer. Math. Soc., Providence, RI; International Press, Boston, MA, 2009.

[16] Kannan, R., Lovász, L., Simonovits, M., Isoperimetric problems for convex bodies and a localization lemma. Discrete Comput. Geom., Vol. 13, No. 3-4, (1995), 541-559.

[17] Klartag, B., Marginals of geometric inequalities. Geometric aspects of functional analysis, Springer Lecture Notes in Math., Vol. 1910, (2007), 133-166.

[18] Klartag, B., Uniform almost sub-gaussian estimates for linear functionals on convex sets. Algebra i Analiz (St. Petersburg Math. Journal), Vol. 19, No. 1, (2007), 109-148.

[19] Klartag, B., Poincaré inequalities and moment maps. Ann. Fac. Sci. Toulouse Math., Vol. 22, No. 1, (2013), 1-41.

[20] Kolesnikov, A. V., On Sobolev regularity of mass transport and transportation inequalities. Theory Probab. Appl., Vol. 57, No. 2, (2013), 243-264.

[21] Kolesnikov, A. V., Hessian metrics, $C D(K, N)$-spaces, and optimal transportation of log-concave measures. Preprint, arXiv:1201.2342

[22] McCann, R. J., A convexity principle for interacting gases. Adv. Math., Vol. 128, (1997), 153-179.

[23] Kallenberg, O., Foundation of modern probability. Second edition. Probability and its Applications (New York). Springer-Verlag, New York, 2002.

[24] Øksendal, B., Stochastic differential equations. An introduction with applications. Sixth edition. Universitext. Springer-Verlag, Berlin, 2003. 
[25] Payne, L. E., Weinberger, H. F., An optimal Poincaré inequality for convex domains. Arch. Rational Mech. Anal., Vol. 5, (1960), 286-292.

[26] Rockafellar, R. T., Convex analysis. Princeton Mathematical Series, No. 28, Princeton University Press, Princeton, NJ, 1970.

[27] Saloff-Coste, L., Aspects of Sobolev-type inequalities. London Mathematical Society Lecture Note Series, Vol. 289. Cambridge University Press, Cambridge, 2002.

[28] Urbas, J., On the second boundary value problem for equations of Monge-Ampère type. J. Reine angew. Math., Vol. 487, (1997), 115-124.

[29] Wang, X.-J., Zhu, X., Kähler-Ricci solitons on toric manifolds with positive first Chern class. Advances in Math., Vol. 188, (2004), 87-103. 